\title{
The radial structure of protostellar accretion disks: influence of jets
}

\author{
C. Combet and J. Ferreira
}

\author{
Laboratoire d'Astrophysique de Grenoble, Université Joseph Fourier, CNRS UMR5571, BP 53, 38041 Grenoble Cedex 9, France \\ e-mail: ccombet@obs.ujf-grenoble.fr
}

Received 25 September 2007 / Accepted 29 November 2007

\section{ABSTRACT}

\begin{abstract}
Context. The radial structure of accretion disks is a fundamental issue regarding star and planet formation. Many theoretical studies, focussing on different aspects such as e.g. disk emissivity or ionisation, have been conducted in the context of the standard accretion disk (SAD) model, where no jet is present.

Aims. We wish to calculate the structure of young stellar object (YSO) accretion disks in an approach that takes into account the presence of the protostellar jets. The radial structure of these jet emitting disks (JED) should then be compared to that of SADs. Methods. The analytical treatment used in this work is similar to standard modelling of accretion disks but uses the parameter space of magnetised accretion-ejection structures that include the jet torque on the underlying disk. In this framework, the analytical expressions of key quantities are derived, such as mid-plane temperatures, surface densities or disk aspect ratios.

Results. We find that JEDs present a structure different from the SADs, which can be observationally tested. The implications on planet formation in the inner regions of accretion disks are briefly discussed. We also supply sets of analytical formulae, valid in different opacity regimes, for the disk quantities. These expressions can be readily used for any work where the disk structure is needed as an input for the model.
\end{abstract}

Key words. accretion, accretion disks - ISM: jets and outflows - stars: formation - method: analytical

\section{Introduction}

Accretion disks are ubiquitous in the Universe. In particular, they are found in active galactic nuclei (AGN), around stellar black holes (X-ray binaries) and in young stellar objects (YSO). From a theoretical point of view, accretion disks have been extensively studied in the context of the standard accretion disk model (hereafter SAD, Pringle \& Rees 1972; Shakura \& Sunyaev 1973; Pringle 1981), be it for AGN or YSO. These early studies were conducted before the discovery of jets and focused on the physics of accretion only. Many refinements have been included to the initial SAD approach over the last thirty years, but the basic idea is still that of these seminal papers: the gas inward motion is ensured by the radial turbulent transport of angular momentum from the inner to the outer parts of the disk. To date, the magnetorotational instability (MRI, Balbus \& Hawley 1991) is the best candidate to provide and sustain the level of turbulence required in accretion disks. For the specific case of YSO - which is our main concern in this work - the standard theory of accretion disks has been widely used to determine their radial and vertical structure. This is a fundamental issue if one is to understand how stars form as well as the initial conditions of planet formation and migration.

After a few decades of observations, it is now clear that disk accretion onto a central object and bipolar ejections cannot be disentangled. Briefly, accretion is believed to power the jets which, in turn, vertically remove part of the disk angular momentum allowing accretion to proceed. This accretion-ejection picture is observed on many astrophysical scales; jets are present in AGN, microquasars, YSO, and have more recently been observed emerging from brown dwarfs (Ferrari 1998; Mirabel \& Rodríguez 1999; Bally et al. 2007; Whelan et al. 2005). Despite the advances in the standard theory of accretion disks, the latter does not provide any explanation to the production of jets.

We will focus on the specific case of YSO. About $30 \%$ of $\mathrm{T}$ Tauri stars (Class 2 objects) present bipolar ejection. This percentage increases to $100 \%$ for Class 0 objects, the earliest stage of star formation. Several models have been developed to explain the jets seen in T Tauri stars. Stellar winds have been invoked (Sauty et al. 2002) and may be present in the inner parts of the jets; however, such winds cannot sustain the observed mass loss rates and cannot therefore be the main engine of the jets (Ferreira et al. 2006a). To date, two accretion-powered wind models exist: i) the X-wind model (e.g. Shu et al. 2000); and the ii) extended disk wind model (e.g. Pudritz \& Norman 1986; Wardle \& Koenigl 1993; Ferreira \& Pelletier 1993). Both models are based on the same mechanism, the so-called magnetorotational launching (originally developed by Blandford \& Payne 1982, for the case of AGN), and only differ from each other in the origin and configuration of the magnetic field threading the disk and the size of the launching region. It will require higher angular resolution observations to have a definite answer regarding the process(es) at play. However, Ferreira et al. (2006a) have gathered indirect evidence (from jet rotation velocities) that appears to favour extended disk-wind theories.

If the jets are indeed accretion powered, which seems to be the case based on our present knowledge, then the jets must affect the structure of the underlying region of the disk powering them. Hence, the SAD model cannot be used in this region where most of the angular momentum is transported away vertically in the jets. In this work, we calculate the radial structure of a jet emitting disk (JED) and compare it to that of a SAD. The paper is organised as follows:

- in Sect. 2, we briefly present the framework of the magnetised accretion ejection structures (MAES). This extended 
disk wind model is a self-consistent description of the accretion disk and the jet it powers. This approach allows us to quantify the effect of the jet on the disk;

- using the key parameters of the previous model, the main equations used to calculate the JED/SAD structure are provided in Sect. 3;

- the results, and in particular a comparison between the two (jet emitting and standard) types of accretion disks, are presented in Sect. 4;

- before concluding, Sect. 5 raises a few issues and focusses on the possible implications of the existence of JED with regard to planet formation and migration.

\section{Jet emitting disks: the MAES framework}

The MAES model (Ferreira \& Pelletier 1993) has been developed so as to treat consistently both the accretion disk and the jet it generates. The idea is the same as in earlier studies of magnetocentrifugally launched disk winds (Blandford \& Payne 1982). In the MAES, however, the solution starts from the midplane of the resistive magnetohydrodynamical (MHD) disk and evolves outwards in the ideal MHD wind/jet. This differs drastically from other studies where the disk was only treated as a boundary condition, thereby forbidding any precise quantification of the effect of the MHD wind on the disk.

\subsection{Properties relevant to this work}

It would be lengthy but also irrelevant to present the MAES model in great detail in this paper. Many papers have dealt with the subject, from both analytical and numerical point of views, and we refer the reader to these papers for further details (e.g. Ferreira 1997; Casse \& Ferreira 2000a,b; Ferreira 2002; Ferreira \& Casse 2004; Casse \& Keppens 2002, 2004; Zanni et al. 2007). Instead, we outline the few key elements of the model that are important to our work.

In a resistive MAES disk, both angular momentum and magnetic field are transported using an alpha prescription. To that end, a local turbulent resistivity ( $v_{\mathrm{m}}$ magnetic diffusivity) is supplemented to the usual turbulent viscosity $v_{\mathrm{v}}$ (used in the standard theory, Shakura \& Sunyaev 1973):

$$
\begin{aligned}
v_{\mathrm{v}} & =\alpha_{\mathrm{v}} \Omega_{\mathrm{K}} h^{2}, \\
v_{\mathrm{m}} & =\alpha_{\mathrm{m}} v_{\mathrm{A}} h,
\end{aligned}
$$

with $\Omega_{\mathrm{K}}$ the Keplerian rotation frequency, $v_{\mathrm{A}}$ the Alfvèn velocity and $h$ the disk half thickness.

Accretion solely depends on the removal of the disk angular momentum. A relevant quantity is then the ratio $\Lambda$ of the jet torque to the viscous torque. This ratio characterises the dominant agent responsible for the extraction of angular momentum. For a $\mathrm{SAD}$, no jet is present: angular momentum is only radially transported by the turbulent viscosity and $\Lambda=0$. However, in a JED, angular momentum is transported vertically in the wind, along the magnetic field lines, and $\Lambda>0$. It has been shown in previous work that steady ejection requires $\Lambda \sim 1 / \epsilon$, with $\epsilon=h / r$ the disk aspect ratio (Ferreira 2002). In general MAES solutions present large values of $\Lambda$ : this emphasises the fact that from the moment a jet is launched, most of the energy and angular momentum will be evacuated in the jets (see Appendix A).

Concerning the transport of matter, the accretion rate in MAES solutions follows $\dot{M}_{\mathrm{a}} \propto r^{\xi}$, where $\xi$ is the ejection efficiency. One of the results of the MAES is that the latter lies in the range $\left[10^{-3}, 5 \times 10^{-1}\right]$ to steadily provide super-Alfvénic jets.
Mass conservation in a MAES, ranging from the inner radius $r_{\text {in }}$ to the outer radius $r_{\mathrm{J}}$ reads $\dot{M}\left(r_{\mathrm{J}}\right)=2 \dot{M}_{\text {jet }}+\dot{M}\left(r_{\text {in }}\right)$ from which one gets,

$$
\frac{2 \dot{M}_{\mathrm{J}}}{\dot{M}\left(r_{\mathrm{J}}\right)}=1-\left(\frac{r_{\mathrm{in}}}{r_{\mathrm{J}}}\right)^{\xi} \simeq \xi \ln \frac{r_{\mathrm{J}}}{r_{\mathrm{in}}} .
$$

The amount of matter being ejected thus depends on the ejection index, but also on the extension of the jet emitting disk. Hence, with the typical values $r_{\text {in }}=0.04 \mathrm{AU}, r_{\mathrm{J}}=0.5 \mathrm{AU}$ and $\xi=0.05, \sim 10 \%$ of the mass will escape into the jets, in agreement with current observational estimates (for more details see Ferreira et al. 2006a).

The more angular momentum is removed the larger the accretion velocity $u_{0}$. The sonic Mach number in the disk $m_{\mathrm{s}} \equiv$ $u_{0} / \Omega_{\mathrm{K}} h$ can be rewritten under the form

$m_{\mathrm{s}} \equiv \frac{u_{0}}{\Omega_{\mathrm{K}} h}=\alpha_{\mathrm{v}} \epsilon+2 q \mu=\alpha_{\mathrm{v}} \epsilon(1+\Lambda)$,

where $\alpha_{\mathrm{v}} \epsilon$ denotes the effect of standard transport, and $2 q \mu$ denotes the specific contribution of the magnetic torque. The magnetisation ${ }^{1} \mu=B^{2} / \mu_{0} P$ measures the strength of the magnetic field in the disk and $q=\mu_{0} J_{r} h / B_{z}$ is the normalised radial electric current density flowing at the disk midplane. This last parameter measures the magnetic shear as it provides an estimate of the toroidal magnetic field component at the disk surface, namely $B_{\phi}^{+} \simeq-q B_{z}$.

In a SAD, $m_{\mathrm{s}}=\alpha_{\mathrm{v}} \epsilon$, with typically $\alpha_{\mathrm{v}}=10^{-2}$ and $\epsilon=h / r<$ 1 , so that the accretion velocity is largely subsonic. However, the situation in a JED is very different as MAES solutions present high accretion velocities with $m_{\mathrm{s}} \sim 1$. This is because steadystate MAES solutions are generally found close to equipartition with $\mu \in[0.1-1]$ and $q$ of the order of unity (Ferreira \& Pelletier 1995; Ferreira 1997). In turn, $q \sim 1$ is only possible for a large level of turbulence, namely $\alpha_{\mathrm{m}} \sim 1$ : for smaller values of the magnetic diffusivity, the toroidal field is much larger and the vertical equilibrium is no more possible. As a consequence the torques ratio reads $\Lambda=2 q \mu / \alpha_{\mathrm{v}} \epsilon \simeq 1 / \epsilon$, where we assumed for simplicity $\alpha_{\mathrm{v}}=\alpha_{\mathrm{m}}$. Note that if the viscous parameter $\alpha_{\mathrm{v}}$ is much smaller than unity, $\Lambda$ becomes even larger.

\subsection{JED - SAD transition}

A schematic representation of the accretion structure we consider here is represented in Fig. 1. The outer parts of the disk have the characteristics of a SAD whereas the inner part is occupied by a JED. The latter has the properties of the MAES as detailed above. The magnetisation $\mu$ provides the criterion for the transition between the two types of disk ${ }^{2}$.

As we indicated previously, a SAD assumes the presence of a turbulent angular momentum transport such that the effective Reynolds number $\mathcal{R}_{\mathrm{e}}=r u_{0} / v_{\mathrm{v}}$ is of order unity. The usual assumption is then a comparable magnetic Reynolds number $\mathcal{R}_{\mathrm{m}}=r u_{0} / v_{\mathrm{m}}$, namely a magnetic Prandtl number of order unity (see e.g. Heyvaerts et al. 1996). If one considers the presence of a large-scale magnetic field threading the disk, such a small value of $\mathcal{R}_{\mathrm{m}}$ translates into straight field lines, i.e. $B_{z} \gg B_{r}^{+}$. The radial distribution $B_{z}(r)$ is provided by the induction equation which describes the interplay between advection and diffusion. Since

\footnotetext{
1 It is directly linked to the usual plasma beta parameter by $\mu=2 / \beta$.

2 This picture has been also successfully applied to X-ray binaries Ferreira et al. (2006b).
} 


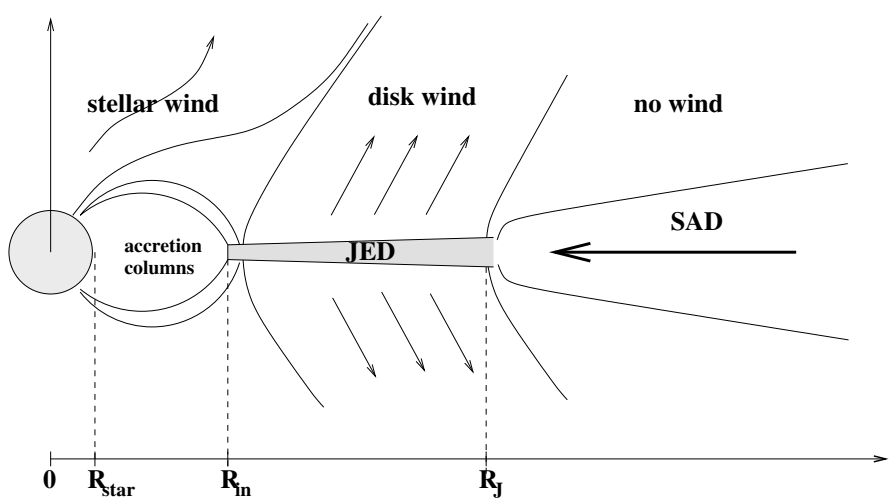

Fig. 1. Sketch of the accretion configuration suggested in this work. The accretion disk is constituted by a SAD in the outer part whereas the inner part is occupied by a JED. See text for details. Accretion columns onto the central object and a possible stellar wind are also represented, although they are not considered in the study.

there is no significant bending in a SAD, this equation writes in steady state,

$\nu_{\mathrm{m}} \frac{\partial B_{z}}{\partial r} \simeq u_{r} B_{z}$

with the obvious exact solution,

$B_{z} \propto r^{-\mathcal{R}_{\mathrm{m}}}$.

Any large-scale magnetic field will thus naturally increase toward the centre in a SAD, as long as MHD prevails. Apart from triggering the MRI, the dynamical importance of this field is in fact measured by the disk magnetisation $\mu(r)$. Therefore, we also need to evaluate the radial distribution of the total pressure $P \simeq \rho \Omega_{\mathrm{K}} h^{2}$. It can be written as:

$P=\frac{\dot{M}_{\mathrm{a}} \Omega_{\mathrm{K}}}{4 \pi r m_{\mathrm{s}}} \propto r^{-5 / 2}$,

where both the accretion rate $\dot{M}_{\mathrm{a}}$ and $m_{\mathrm{s}}=\alpha_{\mathrm{v}} \epsilon$ have been assumed constant ${ }^{3}$. Using Eq. (5), we obtain,

$\mu \propto r^{-\delta}$ with $\delta=2 \mathcal{R}_{\mathrm{m}}-5 / 2$.

Strictly speaking, the magnetisation will increase toward the centre whenever $\mathcal{R}_{\mathrm{m}}>5 / 4$. Thus, unless the magnetic Prandtl number is significantly less than unity, this is a condition that is certainly automatically satisfied in SADs. However, making a transition to a JED requires a large value of $\mu$, namely $\mu \sim 1$ : a JED will therefore exist only below a transition radius $r_{\mathrm{J}}$ such that $\mu\left(r_{\mathrm{J}}\right) \simeq 1$. Below this radius, the radial dependency of the vertical magnetic field is different than that given by Eq. (5). Indeed, a JED requires a field close to equipartition throughout, so $B_{z} \propto r^{-5 / 4+\xi / 2}$ ( $\xi$ is the ejection efficiency).

Not all accretion disks might achieve such a transition as it depends on the magnetisation at the disk's outer edge. This outer boundary condition is itself reminiscent of the history of the protostellar system, that is of both the magnetisation of the parent cloud and the subsequent collapse. It is interesting that, indeed, not all young stars have detectable jets. In our picture, that would be explained by the lack of a JED in the inner-accretion disk. Using a sample of classical T Tauri stars (CTTS), Ménard \& Duchêne (2004) found that CTTS are oriented randomly with

\footnotetext{
${ }_{3}$ Note that $\epsilon$ is only slowly varying with the radius in a SAD around a protostar so that $h \propto r$ is a good approximation.
}

respect to the local interstellar field. This may indicate that interstellar magnetic fields do not play a strong role in enforcing the direction of the final (i.e. stellar) angular momentum. However, sources with strong outflows do have disks mostly perpendicular to the field (i.e. jets are aligned to it as first found by Strom et al. 1986), whereas sources with no jet detected are parallel. That could be a hint that only objects with disks perpendicular to the interstellar magnetic field give birth to magnetised central regions, namely JEDs.

For the purpose of this paper, we will treat $r_{\mathrm{J}}$ as a free parameter and compute the radial structure of JEDs. It is noteworthy that $r_{\mathrm{J}}$ might be observationally determined by measuring the angular velocity in jets (Bacciotti et al. 2002; Pesenti et al. 2004). Taking kinematic constraints from several T Tauri jets, Ferreira et al. (2006a) found that $r_{\mathrm{J}}$ would typically range between 0.2 to a few astronomical units. However, these values must be considered with caution as they might represent upper limits only. Furthermore, we restrict ourselves to the optically-thick case in this preliminary study. This is justified as most CTTS show optically-thick inner disks. Nevertheless, one has to bear in mind the existence of transitional disks (Calvet et al. 2002; D'Alessio et al. 2005; Ratzka et al. 2007): the spectral energy distributions of these disks suggest the presence of large optically thin inner holes, which are generally explained by invoking planet growth in these inner regions. An optically thin JED could be an alternative/complementary interpretation to the large inner gaps they display and this hypothesis deserves further investigation.

There are also some spectroscopic indications that gas is settled in the innermost hot disk regions where dust has been sublimated (Najita et al. 2007). We will not consider line emission in this work, as these inner regions are probably strongly affected by the stellar magnetosphere. We will therefore use, for the disk inner radius $r_{\text {in }}$, an arbitrary, but representative value of the disk truncation radius, $r_{\text {in }}=0.04 \mathrm{AU}$.

\section{Calculation of the disk structure}

We are interested in deriving the radial structure of an accretion disk, be it a SAD or JED. The advantage of the approach used in this work is that both types of disks are described using the same formalism: the only difference lies in the values of the MAES parameter $\Lambda$, thus $m_{\mathrm{s}}$. We make the assumption of a geometrically thin, optically thick steady-state disk, rotating at Keplerian velocity, i.e. with a frequency $\Omega_{\mathrm{K}}=\sqrt{G M_{\star} / r^{3}}$. We also assume that the accretion rate $\dot{M}_{\mathrm{a}}$ does not depend on the distance to the central object. This is justified since solutions to the MAES problem give $\dot{M}_{\mathrm{a}} \propto r^{\xi}$, with $\xi \sim 10^{-3}-5 \times 10^{-1}$ (Casse \& Ferreira 2000b). We also assume the gas and the dust to be well coupled, at the same temperature, and that the mixture behaves as an ideal gas.

\subsection{Basic equations}

We restrict ourselves to the simple case of a steady state accretion disk. The calculation of the disk structure relies on the equality of the cooling and heating terms. The latter can be written in a generic form as

$Q^{+}=f \times \frac{G M_{\star} \dot{M}_{\mathrm{a}}}{8 \pi r^{3}}$

where $f$ represents the fraction of gravitational potential energy that is used to heat the gas. For this work, the gas is only locally heated by viscous effects and any other source of heating, such 
as irradiation from the $\mathrm{star}^{4}$ or dust-gas collisional heating (occurring when $T_{\text {dust }} \neq T_{\text {gas }}$ ) are discarded. In the MAES context, it has been shown (see Appendix A) that

$f=\frac{1}{1+\Lambda}$

- For a $\mathrm{SAD}, \Lambda=0$ and $f_{\mathrm{SAD}}=1$ : in that case, the viscosity is responsible for converting all the mechanical energy into heat.

- For a steady-state JED, however, $\Lambda \sim \epsilon^{-1} \gg 1$ and $f_{\mathrm{JED}} \ll 1$ : as mentioned earlier, when a jet is present, most of the disk energy leaves in the jet as an MHD Poynting flux. Only a small fraction of that energy remains in the disk to be converted into heat and radiated away.

As for the cooling, we assume that the disk radiates like a black body with an effective temperature $T_{\text {eff }}$, which leads to (Hubeny 1990):

$Q^{-}=\sigma T_{\mathrm{eff}}^{4} \approx \frac{3}{8 \tau} \sigma T_{0}^{4}$,

where the optical depth $\tau \approx \kappa \rho_{0} h$ links the effective temperature $T_{\text {eff }}$ to the midplane temperature $T_{0}$ via the opacity of the gas $\kappa$. This link between effective and central temperatures holds only if energy transport is done vertically by photon diffusion. D'Alessio et al. (1998) showed that radiative energy transport was the dominant mechanism in SAD, with respect to convection or turbulent transport. However, the level of turbulence required in a JED $\left(\alpha_{\mathrm{m}} \sim 1\right)$ is much larger than that usually assumed in a SAD $\left(\alpha_{\mathrm{v}} \sim 10^{-2}\right)$ so that the results of D'Alessio et al. (1998) may not apply here. Nevertheless, without any reliable expression for a turbulent energy flux, we restrict ourselves to the usual approximation. For the opacity, we adopt the standard $\kappa=\bar{\kappa} \rho_{0}^{a} T_{0}^{b}$ form, where $\bar{\kappa}, a$ and $b$ have to be adjusted regarding the dominant coolant present in the gas. We use the values given in Bell \& Lin (1994) that includes eight opacity regimes, from dust dominated cooling to electron scattering cooling ${ }^{5}$ (although this regime is not reached by the temperatures at play in YSO disks).

The Bell and Lin opacity adjustments can be used to derive the disk structure analytically, which is not the case with tabulated results. The value of the opacity of the gas at a given temperature and density is a fundamental quantity regarding the calculation of an accretion disk structure and should be evaluated carefully. Many authors have numerically calculated Rosseland mean opacities, using different models (Alexander \& Ferguson 1994; Henning \& Stognienko 1996; Semenov et al. 2003). The Bell \& Lin prescription deviates from more refined approaches around $1500-1800 \mathrm{~K}$, where it significantly underestimates $\kappa$ (Semenov et al. 2003). We stopped our disk calculations whenever the central temperature reached this value. For all JED models, the inner disk edge $r_{\text {in }}$ is achieved at lower temperatures (see Fig. 2). However, SAD models are affected and this is the reason why, in that case, the disk inner radius is actually larger than the chosen $r_{\text {in }}$ (see also Papaloizou \& Terquem 1999). More refined SAD calculations should be performed at these inner radii, probably taking into account dust sublimation and line emission from the gas component. This is beyond the scope of the present paper.

\footnotetext{
4 This hypothesis is further discussed in Sect. 5.1.

5 In Appendix B, we give the general expressions of the radial structure of a JED, keeping $\bar{\kappa}, a$ and $b$ as free parameters.
}

\subsection{Radial structure of the accretion disk}

A standard way of writing the vertical hydrostatic equilibrium of a disk is (e.g., Papaloizou \& Terquem 1999)

$\frac{\partial P}{\partial z}=-\rho \Omega_{\mathrm{K}}^{2} z$

Integrated over the thin disk, the hydrostatic equilibrium defines the scale height of the disk $h$, via the aspect ratio $\epsilon=h / r$ :

$\epsilon^{2}=\frac{P_{0}}{\rho_{0} \Omega_{\mathrm{K}}^{2} r^{2}}$,

where quantities with the subscript 0 , refer to midplane values. Using the perfect gas equation of state, the previous equation reads:

$\epsilon^{2}=\frac{k_{\mathrm{B}} T_{0}}{\bar{\mu} m_{\mathrm{p}} \Omega_{\mathrm{K}}^{2} r^{2}}$,

where $k_{\mathrm{B}}$ is the Boltzmann constant, $\bar{\mu}$ the mean molecular weight of the gas, and $m_{\mathrm{p}}$ the proton mass.

Defining $u_{0}$ to be the inward radial velocity of the flow, one can simply calculate the density as

$\rho_{0}=\frac{\dot{M}_{\mathrm{a}}}{4 \pi r h u_{0}}=\frac{\dot{M}_{\mathrm{a}}}{4 \pi \Omega_{\mathrm{K}} r^{3}} \frac{1}{m_{\mathrm{s}} \epsilon^{2}}$,

where $m_{\mathrm{s}}$ is the sonic Mach number, defined by Eq. (3) in the MAES model.

Equalling the heating and cooling terms

$Q^{+}=Q^{-}$

and using Eqs. (11) and (12) it is possible to express all the thermodynamical quantities (in particular the midplane temperature $T_{0}$ and the disk surface density $\Sigma=2 \rho h$ ) as a function of the radius and of the parameters of the problem, namely under the generic form,

$X(r) \propto \mathcal{A}_{x}\left(M_{\star}, \dot{M}_{\mathrm{a}}, m_{\mathrm{s}}, \bar{\kappa}, a, b\right) r^{\delta_{x}}$,

where $\mathcal{A}_{x}$ and $\delta_{x}$ relates to the quantity $X$ and depends on the problem parameters. The explicit forms of these quantities can be very lengthy and are postponed to Appendix B for the sake of legibility. Note that these expressions could be directly used by those wishing to quickly derive the structure of a JED for a given set of stellar and opacity parameters.

Concerning the magnetic field and as stressed in Sect. 2.1, the necessary condition for launching a self-collimated jet from a Keplerian accretion disk is the presence of a large-scale vertical magnetic field close to equipartition $(\mu \sim 1$, Ferreira \& Pelletier 1995). This condition allows us to estimate the strength of the magnetic field

$B_{z} \simeq 0.2\left(\frac{M_{\star}}{M_{\odot}}\right)^{1 / 4}\left(\frac{\dot{M}_{\mathrm{a}}}{10^{-7} M_{\odot} / y r}\right)^{1 / 2}\left(\frac{r}{1 \mathrm{AU}}\right)^{-5 / 4+\xi / 2} \mathrm{G}$.

For the specific case of our calculation, the ejection efficiency $\xi=0$. This equation along with the set provided in Appendix B $\left(T_{0}, \epsilon\right.$ and $\Sigma$ ) completely determine the disk structure.

\section{Results}

The main features of jet emitting disk can now be derived from the expression collected in Appendix B. In Sect. 4.1, the radial variations of the key quantities of the JED are presented. Comparison to the standard case is also drawn. In Sect. 4.2, we illustrate the effect of a jet emitting region of the disk on the spectral energy distribution. 

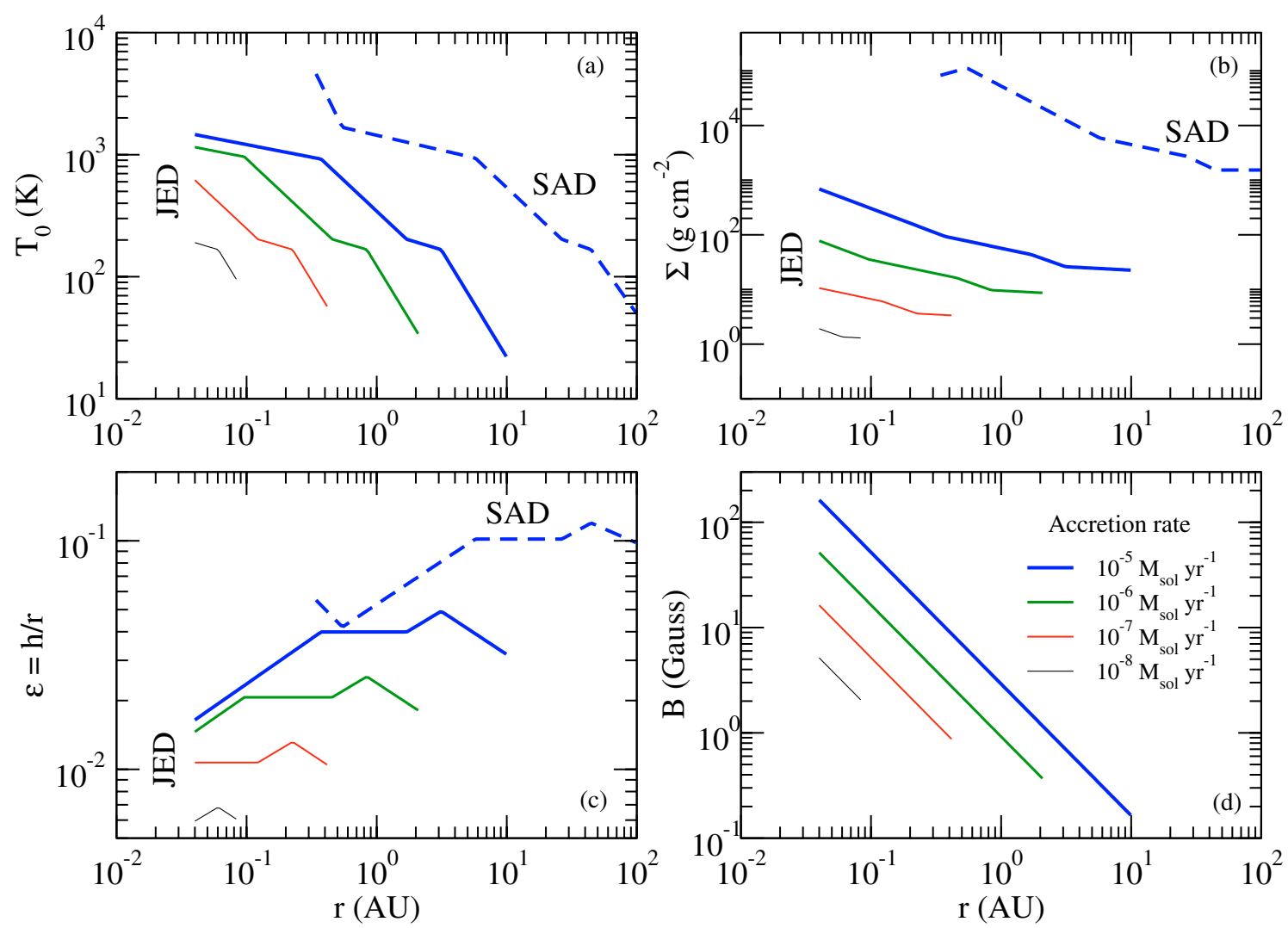

Fig. 2. Radial variation of key disk quantities. Upper-left: midplane temperature. Upper-right: surface density. Lower-left: aspect ratio of the disk. Lower-right: large scale vertical magnetic field intensity. Line thickness represents the increasing accretion rates considered for the JED: $\dot{M}_{\mathrm{a}}=10^{-8}, 10^{-7}, 10^{-6}$ and $10^{-5} M_{\odot} \mathrm{yr}^{-1}$. However, the SAD case is computed for $\dot{M}_{\mathrm{a}}=10^{-5} M_{\odot} \mathrm{yr}^{-1}$ and $\alpha_{\mathrm{v}}=0.01$ only in order to prevent overlapping curves.

\subsection{JED: main characteristics}

In Fig. 2 the radial dependences of the central temperature (upper-left), aspect ratio (lower-left), surface density (upperright) and magnetic field intensity (lower-right) of the JED are plotted in solid lines, as given by Eq. (B.1)-(B.3) and (15). The quantities - but for the magnetic field - appear as broken powerlaws, each segment corresponding to a given opacity regime $(\bar{\kappa}, a, b)$. The transition radius between two regimes of the Bell \& Lin opacity, say $i$ and $i+1$, are obtained by setting $\kappa_{i}(r)=\kappa_{i+1}(r)$, and solving for $r$.

The disk quantities have been plotted for different mass accretion rates, from $10^{-8}$ to $10^{-5} M_{\odot} \mathrm{yr}^{-1}$. In agreement with intuition, the higher the accretion rate, the higher the temperature, surface density, and disk aspect ratio. Note also that more opacity regimes need to be taken into account for the highest accretion rates as a result of the increasing the temperature. We have truncated each curve at the outer radius for which the gas becomes locally optically thin to its own radiation and where our description of the radiation in Eq. (10) stops being valid. But since accretion disks of CTTS are optically thick, this radius marks an upper limit for $r_{\mathrm{J}}$. Figure 3 is another representation of the latter point and gives in the $\left(r, \dot{M}_{\mathrm{a}}\right)$ plane, the region where the optical depth $\tau$ is greater than unity in a JED. There are not many sources where both the accretion rate and $r_{\mathrm{J}}$ have been estimated. This is however the case for DG Tau $\left(\dot{M}_{\mathrm{a}} \sim 2 \times 10^{-6} M_{\odot} \mathrm{yr}^{-1}\right.$, Bacciotti et al. 2002) for which Ferreira et al. (2006a) estimated $r_{\mathrm{J}} \sim 1.5-4.5$ AU: these values fall into the validity range of our calculation given in Fig. 3.

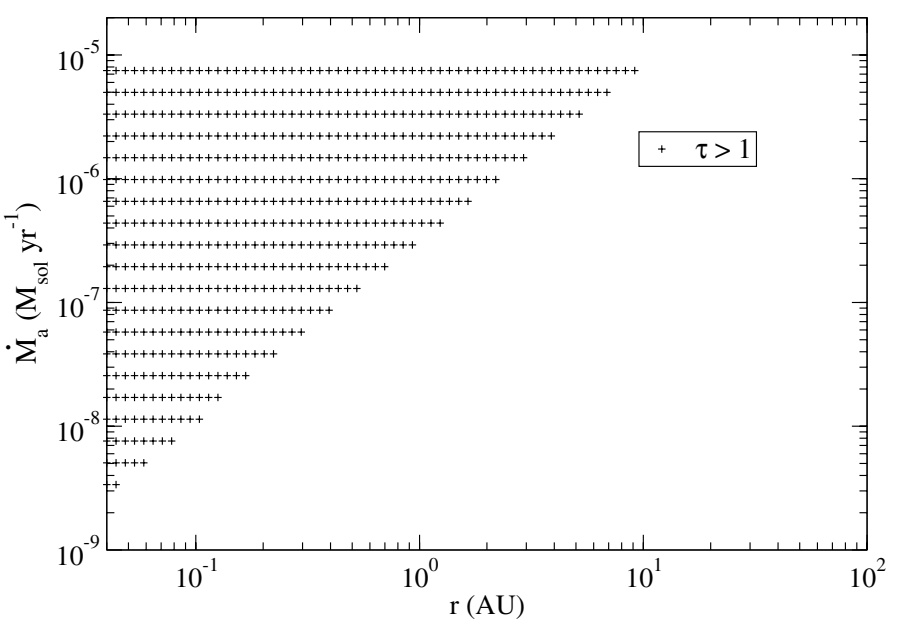

Fig. 3. Region of optically thick JED in the $(r, \dot{M})$ plane. Crosses represent the validity range of our calculation.

Coming back to Fig. 2, all quantities (except for the magnetic field) are compared to the SAD case (dashed curve). For the latter, we have plotted only the highest accretion rate (and use $\alpha_{\mathrm{v}}=10^{-2}$ ) to prevent overlapping curves. Also not shown here, our SAD models are in agreement with the disk structure derived in Papaloizou \& Terquem (1999). Curves for smaller values of the accretion rate and other $\alpha_{\mathrm{v}}$ are available in that paper.

The radial structure of a JED is found different than that of its equivalent SAD: at a given accretion rate, the JED is cooler (Fig. 2a), lighter (Fig. 2b), and thinner (Fig. 2c) than the SAD. 
In particular, at a given radius, the surface density can vary by $\sim$ two orders of magnitude. This implies a large density jump at the transition radius $r_{\mathrm{J}}$, that remains whatever the chosen value of the turbulent parameter $\alpha_{\mathrm{v}}$. Implications of this point are further discussed in Sect. 5.2.

Turning to the magnetic field, one might object that fields of the strength given by Eq. (15) and illustrated in Fig. $2 \mathrm{~d}$ are impossible in accreting systems. However, the value of this magnetic field is actually far smaller than the one estimated from the interstellar magnetic field assuming either ideal MHD $B \propto n$, or $B \propto n^{1 / 2}$ (Heiles et al. 1993; Basu \& Mouschovias 1994). If we take the fiducial values $n \sim 1 \mathrm{~cm}^{-3}$ and $B \sim 4 \mu \mathrm{G}$ observed within dense clouds and use the law $B \propto n^{1 / 2}$ (Crutcher 1999), we get a magnetic field at $1 \mathrm{AU}$ ranging from 10 to $10^{3} \mathrm{G}$ (depending on the density). The main problem is then to get rid of the magnetic field during the infalling stage. This issue is still in debate. It seems nevertheless straightforward to build up accretion disks threaded by an intense large-scale magnetic field from 3D collapse calculations (see e.g. Banerjee \& Pudritz 2006).

The observation of magnetic fields is a difficult task and no measurements have been obtained so far in a disk known to drive a jet. However, Donati et al. (2005) have managed to measure the magnetic field strength in the disk of FUOr, a strong accreting object with $\dot{M}_{\mathrm{a}} \sim 10^{-5} M_{\odot} \mathrm{yr}^{-1}$. Using the spectropolarimeter ESPadOnS, they found a value of $B \sim 1 \mathrm{kG}$ at $0.05 \mathrm{AU}$ from the star. At this distance and for this accretion rate, Eq. (15) gives $B \sim 100 \mathrm{G}$. The magnetic field present at the inner disk of FUOr is actually higher than equipartition: according to the MAES theory, no steady state self-collimated jet can be launched from this disk, which is indeed consistent with observations. If one takes this observation at face value, then one possible explanation is that the disk magnetic flux has been advected and compressed toward the star by the sudden rise of disk accretion rate. This conjecture deserves further investigation. Note also that the presence of a strong (i.e. larger than equipartition) vertical magnetic field in an accretion disk triggers nonaxisymmetric instabilities and spiral waves (Tagger et al. 1992). If these waves bounce back at the inner-disk boundary then a standing spiral pattern is formed and leads to the formation of a magnetised vortex (a MHD Rossby wave) localised at the radius where the Keplerian rotation coincides with the wave frequency (Tagger \& Pellat 1999; Caunt \& Tagger 2001; Stehle \& Spruit 2001). Although the field structure derived by Donati et al. (2005) shows a remarkably high degree of axisymmetry, one might indeed expect nonaxisymmetric perturbations in strongly magnetised disks. However, for the physical conditions envisioned in JEDs, namely a field close to but smaller than equipartition, such instabilities are weak (see however Keppens et al. 2002, for MHD instabilities at $\mu \sim 1$ ).

\subsection{Spectral energy distributions}

The physical properties of a jet-emitting disk are different from those of a standard disk. As a consequence, their radiative properties should also differ and are investigated hereafter. Spectral energy distributions (SED) are one of the main ways to diagnose disks through observations. If the radial structure of the disk does not depend too drastically on the irradiation of the central object (see Sect. 5.1), its radiative properties certainly do (see Dullemond et al. 2007, for a review). The simplest approach considers that stellar illumination can create a super-heated layer at the surface of the disk which, in turn, affects the disk SED (Chiang \& Goldreich 1997; Dullemond et al. 2001). The success of this model comes from both its simplicity (compared to that of a full radiative transfer treatment), and its ability to reproduce observations of TTauris and Herbig Ae/Be stars.

Our purpose here, however, is to compute the SED to illustrate a possible effect of the JED rather than reproduce specific observations. For that reason, the irradiation of the central star on the disk will be included in the crude fashion detailed below.

We will consider two components to the irradiating flux: the stellar radiation and the UV accretion luminosity. In the now widely-accepted magnetospheric accretion model, matter is channelled along the stellar magnetic dipole from the disk onto the star (see Bouvier et al. 2007, and references therein) where it creates the observed hot spots/rings. The UV radiation from these accretion shocks can then illuminate and heat the disk. In the following, $L_{\mathrm{UV}}$ will refer to the UV luminosity of one of the two hot rings, and $L_{\star}$ to the stellar component. Gullbring et al. (1998) estimated from a sample of TTauri stars that,

$2 L_{\mathrm{UV}} \sim \frac{L_{\mathrm{acc}}}{3.5}$

where the total accretion luminosity scales with the accretion mass rate as,

$L_{\mathrm{acc}} \approx \frac{G M_{\star} \dot{M}_{\mathrm{a}}}{R_{\star}}\left(1-\frac{R_{\star}}{r_{\mathrm{in}}}\right)$.

The stellar luminosity is given by

$L_{\star}=4 \pi R_{\star}^{2} \sigma T_{\star}^{4}$,

where $R_{\star}$ and $T_{\star}$ are respectively the star radius and temperature. The irradiation flux $Q_{\text {irr }}$ is then obtained by,

$Q_{\text {irr }}=\frac{L_{\star}+L_{\mathrm{UV}}}{4 \pi r^{2}} \cos \psi$,

with $\psi$ the angle between the incident radiation and the normal to the disk surface. We will assume a fixed incident angle, $\cos \psi=$ 0.05 (i.e., incident angle $\sim 3 \mathrm{deg}$.): this is of course not consistent with our disk model, as $h / r \neq$ const. (Fig. 2 c), but has the benefit of giving a quick estimate of the irradiation component.

With these ingredients, the disk effective temperature required by SED calculation is obtained by

$\sigma T_{\mathrm{eff}}^{4}=Q^{+}+Q_{\mathrm{irr}}$

The viscous heating flux $Q^{+}$is given by Eq. (8) - this flux is much smaller in a JED than in a SAD.

To actually compute the SED, we consider the disk configuration presented in Fig. 1, where a JED is present from $r_{\text {in }}$ to $r_{\mathrm{J}}$ and a SAD from $r_{\mathrm{J}}$ to $r_{\text {out }}$. We choose arbitrarily $r_{\mathrm{J}}=0.5 \mathrm{AU}$ (see e.g. Ferreira et al. 2006a). For illustrative purposes, Fig. 4a gives the disk scale height as a function of the radius. The thick solid line corresponds to the disk studied here, with a transition in $r_{\mathrm{J}}$. The SED obtained from this structure is then compared to the case where a standard disk is present on the entire spatial domain $\left[r_{\text {in }}, r_{\text {out }}\right]$. Using the temperature derived in Eq. (19), the disk SED is computed for a one-solar mass star with the simplest geometry, where the disk is viewed pole-on,

$$
\begin{aligned}
v L_{v}^{\text {disk }} & \equiv 4 \pi d^{2} v F_{v} \\
& =8 \pi^{2} v \int_{r_{\text {in }}}^{r_{\text {out }}} r B_{v}\left(T_{\text {eff }}\right) \mathrm{d} r \\
& =8 \pi^{2} v\left(\int_{r_{\text {in }}}^{r_{\mathrm{J}}} r B_{v}\left(T_{\mathrm{JED}}\right) \mathrm{d} r+\int_{r_{\mathrm{J}}}^{r_{\text {out }}} r B_{v}\left(T_{\mathrm{SAD}}\right) \mathrm{d} r\right) .
\end{aligned}
$$



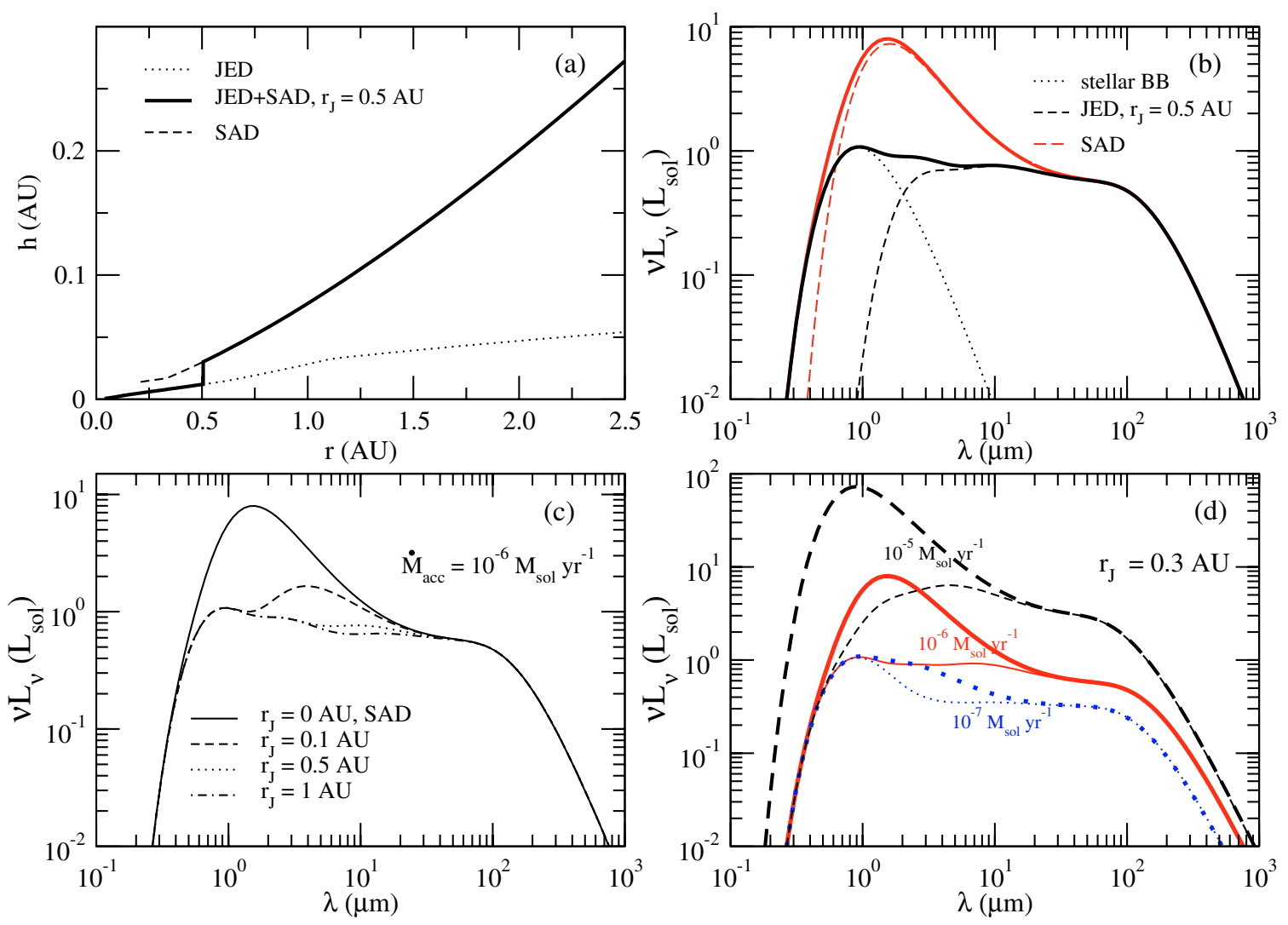

Fig. 4. Upper-left: disk scale height as a function of the distance to the central object. The JED/SAD transition radius is $r_{\mathrm{J}}=0.5 \mathrm{AU}$. Upperright: pole-on viewed spectral energy of the JED+SAD disk of a). Dashed lines correspond to the SAD (thin red) and JED+SAD (thick black) configurations. A $4000 \mathrm{~K}$ stellar blackbody is included (dotted line). The sum of the stellar and disk SED is plotted in thick solid curves (red and black). Lower-left: variation of the total JED+SAD energy distribution with increasing transition radius. Lower-right: influence of the accretion rate on the SED for a fixed transition radius. Thick lines correspond to the SAD alone and thin ones to the JED+SAD case.

In this expression, Planck's law $B_{v}$ depends on the radius through the disk effective temperature $T$. The contribution of the central object to the SED is also considered, using $T_{\star}=4000 \mathrm{~K}$ and $R_{\star}=2.5 R_{\odot}$, and assuming it radiates like a spherical blackbody:

$v L_{v}^{\mathrm{star}}=4 \pi^{2} R_{\star}^{2} v B_{v}\left(T_{\star}\right)$.

The spectral energy distribution is plotted in Fig. $4 \mathrm{~b}$ from infrared to millimetre wavelengths. The dotted and dashed curves correspond respectively to the stellar and disk contributions. The total SED is plotted in thick solid curves, for each disk configuration - SAD alone or JED+SAD. The effect of a jet-emitting region in the disk is characterised by a smaller flux and a redshifted disk SED. Note that Kuncic \& Bicknell (2007) recently reached a similar conclusion more specific to the case of AGN. Although they used similar ingredients to our study, their outflow model was purely phenomenological and did not rely on a physically consistent underlying model (such as the MAES model) for the quantification of the jet torque. This effect increases with the size of the transition radius $r_{\mathrm{J}}$ : this is shown in Fig. 4c, where the discrepancy between the standard SED and the JED+SAD becomes larger for larger $r_{\mathrm{J}}$. This is as expected as it was shown in the previous section that JED were lighter and cooler than their equivalent SAD.

The last effect on the SED is shown in Fig. 4d. For a fixed transition radius $r_{\mathrm{J}}=0.3 \mathrm{AU}$, we compute the SAD and JED +SAD spectral energy distributions for several mass accretion rates, namely $10^{-5}, 10^{-6}$ and $10^{-7} M_{\odot} \mathrm{yr}^{-1}$. For a given line pattern, the thickest line corresponds to the standard disk alone.
For the highest accretion rate, the standard disk SED completely overwhelms the stellar component, which explains why the thick dashed curve does not join the others at small wavelengths.

We highlight two interesting effects in this figure. First of all, we find that the discrepancy between the standard and JED+SAD energy distributions is higher, for higher accretion rates: at $10^{-6} M_{\odot} \mathrm{yr}^{-1}$ (and for that particular $r_{\mathrm{J}}$ ), the standard flux is at most 10 times larger than the JED+SAD flux, whereas this value falls to 2.5 at $10^{-7} M_{\odot} \mathrm{yr}^{-1}$. The second effect, linked to the first, concerns the location of this maximum discrepancy. It is found around $1.5 \mu \mathrm{m}$ at $10^{-6} M_{\odot} \mathrm{yr}^{-1}$ and $\sim 3 \mu \mathrm{m}$ at $10^{-7} M_{\odot} \mathrm{yr}^{-1}$. These two points may be relevant regarding observational efforts on the matter. However, these SED are presented to highlight a potential effect only. We must work further to reach a real quantification of this effect when taking properly into account the central object heating of the disk surface.

\section{Discussion}

\subsection{Illumination from the central star}

In the previous section, we included irradiation of the central object in the calculation of the SED as it is known to have a strong influence on the latter. Its influence onto the disk structure however, is less-well established. For that reason, but also for sake of simplicity, the irradiation from the central star was not taken into account in our calculation of the disk structure. Nevertheless, as it decreases in $r^{-2}$ compared to the $r^{-3}$ of the viscous heating Eq. (8), it is expected to become dominant beyond a certain 
distance from the central object, geometrical effects put aside. Dubus et al. (1999) emphasised that the appropriate criterion to check wether the disk structure is dominated by viscous heating is

$$
\frac{Q_{\text {irr }}}{\tau_{\text {tot }}}<Q^{+},
$$

where $Q_{\text {irr }}$ is the irradiation flux coming from the star (see also Rafikov \& De Colle 2006). The total optical depth of the disk $\tau_{\text {tot }}$ appears as the irradiation mainly affects the surface of the disk whereas the viscous heating is assumed to be present on the entire disk thickness.

We have determined the irradiation flux in the previous section, by Eq. (18) and can readily be used with Eq. (8) to check the validity of our disk structure calculation given by the above criterium. For this calculation, however, we will make no assumption about the incident angle for the radiation, but compute it consistently given the disk structure we previously derived. The source of the radiation is considered point-like. This is a reasonable assumption for the UV hot spot located at the end of the accretion column. But it is questionable for the stellar component: the star is an extended source, at least for the most inner radii. Again, for simplicity, we will consider the two components (stellar and UV) as coming from one unique location. By changing the altitude of the spot, we should be quite conservative in our conclusions.

From geometrical considerations, one gets

$$
\cos \psi=\frac{h}{\sqrt{r^{2}+\left(z_{\mathrm{sh}}-h\right)^{2}}}\left[\frac{\mathrm{d} \ln h}{\mathrm{~d} \ln r}-1+\frac{z_{\mathrm{sh}}}{r}\right] .
$$

In the previous expression, all quantities have their usual meaning and $z_{\mathrm{sh}}$ is the light spot altitude. If $z_{\mathrm{sh}}=0$, the standard expression of Frank et al. (2002) is recovered.

Within the JED, we found that when $z_{\text {sh }}$ equals zero, the illumination could safely be discarded for any accretion rate $\dot{M}_{\mathrm{a}} \gtrsim 3 \times 10^{-7} M_{\odot} \mathrm{yr}^{-1}$ (at any radius). The situation changes when the hot spot is located at a higher altitude as it can strike the disk more directly in the inner regions. For $z_{\mathrm{sh}}=0.7 R_{\star}$, i.e. an accretion column starting at the star at a colatitude of $45^{\circ}$, we found that Eq. (22) was not satisfied for $\dot{M}_{\mathrm{a}} \lesssim 7 \times 10^{-7} M_{\odot} \mathrm{yr}^{-1}$. To obtain these thresholds, we required Eq. (22) to be true on the entire radius range. Note that these accretion rates could be lowered to some extent if one only requires the condition to be realised on most of the radius range: it could be argued that a narrow, irradiation-dominated region will not change much in the overall structure of the disk. Basically, illumination of the JED can be neglected at any radius for $\dot{M} \gtrsim$ a few $10^{-7} M_{\odot} \mathrm{yr}^{-1}$. This threshold is consistent with accretion rates of sources driving powerful (detected) jets. Nevertheless, it would be of interest to extend this work to the lower accretion rates by including irradiation in the heating term ${ }^{6}$. From these considerations, we are left confident with the validity of our simplified analytical description, at least for $\dot{M} \gtrsim$ a few $10^{-7} M_{\odot} \mathrm{yr}^{-1}$.

\subsection{Implications for planet formation and migration}

The initial conditions for planet formation depend on the disk's physical properties. We have shown in the previous section that these properties are different from a JED to a SAD. We discuss, hereafter, two of the possible consequences that an accretion structure, such as that of Fig. 1, may have on planet formation.

\footnotetext{
6 This can only be done iteratively (hence, not analytically) as knowledge of the disk structure is required for the determination of $\cos \psi$.
}

Gammie (1996) was the first to present the idea of layered accretion disks where the upper part of the disk is ionised via collisions, cosmic rays, or X-rays - and some embedded inner part stays neutral. The latter, termed dead zone, is then decoupled from the magnetic field, hence from the MRI-induced turbulence, and remains quiescent. Angular momentum is poorly transported outward in the dead zone and no accretion occurs in this region of the disk.

We have shown in the previous section that JEDs were both thinner and lighter than standard disks. As a consequence, they are likely to be more ionised than SAD, as X-ray radiation from the central star and cosmic rays should more deeply penetrate them. The calculation of the actual ionisation degree of JED is postponed to a forthcoming study, which should give a definite answer to the possibility of a dead zone in a JED. However, it is of interest to briefly mention a few issues related to that matter.

Both low and high mass planet formation start with the growth of planetesimals, as well as the necessity of dust settling and agglomeration. The effect of turbulence on agglomeration is still being debated. Indeed, it might help to form planetesimals by trapping dust within turbulent vortices (e.g. Barge \& Sommeria 1995). On the other hand, recent numerical simulations of a fully-turbulent disk showed that only the larger grains (with typical sizes from 1 to $10 \mathrm{~cm}$ ) do settle toward the midplane, whereas the smaller ones stay in suspension in the disk (Fromang \& Papaloizou 2006). They found the thickness of the dust subdisk to be large $(\sim 0.2 \mathrm{~h})$, and also showed that the dust subdisk is thinner (more compact) when a dead zone is taken into account in the simulations. Along the same line, Ciesla (2007) stressed that the efficiency of dust settling and coagulation in the presence of a dead zone was enhanced compared to that of an ionised (i.e., turbulent) disk. From those considerations, layered accretion disks appear to be a privileged location for grain growth and planetesimal formation. If JEDs are indeed fully ionised, hence turbulent, then it is unlikely that they can host the earliest stages of planet formation.

On the other hand, the maximum value of the JED-SAD transition radius $r_{\mathrm{J}}$, as determined by the transition to the opticallythin JED regime, is rather small (see Fig. 3). It reaches $\sim 10 \mathrm{AU}$ only for high accretion rates, found only in embedded sources (Class 0 or I). For accretion rates of some $10^{-8} M_{\odot} \mathrm{yr}^{-1}$, more typical of CTTS, $r_{\mathrm{J}}$ cannot exceed 0.1-0.3 AU. As the JED has a low column density $\Sigma$ (see Fig. 2b), it does not provide ideal conditions for the core accretion process leading to massive planet formation.

Planet migration in a gaseous disk surrounding a central star (Goldreich \& Tremaine 1980) has been a long-standing issue regarding planetary formation theories. The transfer of angular momentum between the planet and the gaseous disk generally results in the inward motion of the planet. This general mechanism affects both low (type I migration) and high mass planets (type II migration) - see (Armitage 2007) for a review and is generally invoked to explain the existence of hot Jupiters. However, the existence of giant planets far from their central star (a few AU) is a real issue. Indeed, type I migration is known to be a fast process and should not allow enough time for core accretion (build up of a massive solid core), i.e. for the existence of giant planets at such distances. Hence, finding a way to slow down or halt type I migration is of importance if one is to understand planetary system formation.

In that respect, Masset et al. (2006) studied the effect of a sudden radial surface density decrease (going inwards) in the disk on type I planet migration. These authors found that such a density jump could indeed trap a protoplanet at the location of 
the transition by a balance between the corotation and Lindbald torques. As shown previously, the surface density of a SAD is always larger than that of a JED for a given accretion rate (see Fig. 2b). Hence, a transition from an outer SAD to an inner JED, as described in Sect. 2.2, would naturally provide the surface density jump necessary to a planet trap. It is therefore likely that planetesimals should first form in the outer, denser SAD and then migrate toward the centre to be halted at the JED-SAD transition.

One must be cautious because in Masset et al. (2006) the physics of the JED and, in particular the presence of the largescale magnetic field, was not taken into account. It would be useful, as a second step, to include this critical ingredient and determine precisely how will be the JED affected by the presence of a protoplanet.

\subsection{The $\dot{M}_{a}-M_{\star}$ relationship in the JED model}

One of the striking observational properties of T Tauri stars and brown dwarfs is the apparent steep correlation between the mass of the central object and the disk accretion rate, namely

$\dot{M}_{\mathrm{a}} \propto M_{\star}^{\alpha}$,

with $\alpha$ lying between 1 and 2 (Muzerolle et al. 2003; Calvet et al. 2004; Alexander \& Armitage 2006; Hartmann et al. 2006). Though seemingly robust observationally - four orders of magnitude in accretion rates and two in mass - the physical origin of this relation is still debated. Padoan et al. (2005) evoked the possibility of a star-disk accreting from a large scale envelope at the Bondi-Hoyle rate, giving precisely $\dot{M}_{\mathrm{a}} \propto M_{\star}^{2}$. However, this scenario does not take into account the angular momentum of the infalling gas. In an other approach, Dullemond et al. (2006) showed how taking into account the imprint of the physical properties of the parent core onto the star-disk system leads to $\dot{M}_{\mathrm{a}} \propto M_{\star}^{1.8}$. Hartmann et al. (2006) explored several mechanisms, but concluded on a word of caution: these authors noted that, if the bulk of the data is well represented by $\dot{M}_{\mathrm{a}} \propto M_{\star}^{2}$, the TTauri stars with the highest accretion rates appear to have $\dot{M}_{\text {a }} \propto M_{\star}$. In that sense, Hartmann et al. (2006) stated that it may not be relevant to look for a universal $\dot{M}_{\mathrm{a}}-M_{\star}$ relationship, as different mass regimes may have different dominating processes. Nevertheless, we believe it is of interest to see what $\dot{M}_{\mathrm{a}}-M_{\star}$ relationship is predicted by the JED model.

The disk accretion rate $\dot{M}_{\mathrm{a}}$, as derived from observations, is actually indicative of the mass flow at the inner disk regions, hence representative of the JED rather than of the outer SAD. But in a JED accretion is achieved through angular momentum removal in jets. This is made possible only because of the presence of a large scale disk magnetic field $B_{\text {disk. }}$. As argued in Sect. 2.2, this field is probably the parent cloud core magnetic field $B_{\text {core }}$ that has been advected and concentrated by the infalling material during the collapse. Such a collapse is induced when the ratio of the magnetic flux to the total mass $\Phi / M_{\text {core }}$ reaches a critical value. This translates into

$B_{\text {core }} \propto M_{\text {core }}$

as an initial condition. Now, assuming that the ratio $\Phi / M$ remains constant (or varies only slowly) during the collapse, one gets

$B_{\text {disk }} \propto M_{\star}$

since most of the infalling cloud core mass ends up in the central star (ejection will not change the relation $M_{\star} \propto M_{\text {core }}$ ). Note that another way to obtain a relation of this kind is by writing
$B_{\text {disk }} \propto B_{\text {core }}^{\eta}$, where the value of the exponent $\eta$ should be provided by full, $3 \mathrm{D}$ collapse calculations. However, we expect it to be close to unity, which would lead to Eq. (26) as well.

The disk magnetic field required in a JED is given by Eq. (15) and leads to

$B_{\text {disk }} \propto \dot{M}_{\mathrm{a}}^{1 / 2} M_{\star}^{1 / 4}$

Inserting this relation into Eq. (26) provides then the $\dot{M}_{\mathrm{a}}-M_{\star}$ relationship expected in JEDs, namely

$\dot{M}_{\mathrm{a}} \propto M_{\star}^{3 / 2}$

If our crude derivation gives a result consistent with the observations, one must however remain cautious: for a given stellar mass $M_{\star}$ there is observationally a large dispersion in $\dot{M}_{\mathrm{a}}$. This has led Clarke \& Pringle (2006) to point out a possible incompleteness of the samples used, which may have led to such an apparent correlation.

\section{Conclusions}

There has been growing observational evidence that the bipolar ejections of matter occurring during the formation of a star are directly linked to the accretion process. From that point of view, jets should have an influence on the structure of the region of the disk that is launching them.

Using the framework of the MAES, we derived the radial structure of such a JED, and compared it to the equivalent SAD. We found that a JED is cooler, thinner, and lighter than a SAD at the same accretion rate. Invoking the idea of a radial transition from an inner JED to an outer SAD, we have also shown in a crude approach that such a transition has an effect on the spectral energy distribution: the flux coming from a JED is smaller and redshifted compared to the SAD. A more detailed study is needed to provide realistic observational predictions.

Several implications on planet formation have also been drawn from the existence of JEDs. On the one hand, and although this needs confirmation, dead zones may not exist in JEDs as their small thickness and low density will favour ionisation. Recent numerical works show that dust settling could be made more difficult in the absence of a dead zone. This raises some doubts regarding the possibility for a JED to host the earliest stages of planet formation. On the other hand, the surface density jump occurring in a JED/SAD transition could serve as a planet trap and halt type I migration.

Protostellar jets have been observed and theoretically studied by the magnetocentrifugal approach (among others) for almost 30 years. Nevertheless, little attention has been paid to their potential effects on the disk structure. In this paper, we stress that these effects indeed exist and further work is to be undertaken for better quantification and observational perspective.

Acknowledgements. The authors wish to thank the referee, C. Dullemond, for his many suggestions that helped us improving the manuscript. Also, C.C. wishes to thanks Dr. Fabio De Colle for useful discussions on irradiated disks. The present work was supported in part by the European Community Marie Curie Actions - Human Resource and Mobility within the JETSET (Jet Simulations, Experiments and Theory) network under contract MRTN-CT-2004 005592.

\section{Appendix A: MAES global energy budget}

The global energy budget of a MAES writes (Ferreira \& Pelletier 1993; Casse \& Ferreira 2000b)

$P_{\text {acc }}=2 P_{\text {jet }}+2 P_{\text {rad }}$ 
where the accretion power, namely the power released by the accreting flow in the disk

$P_{\mathrm{acc}} \simeq \frac{G M_{\star} \dot{M}_{\mathrm{a}, \mathrm{J}}}{2 r_{\mathrm{in}}}\left[\left(\frac{r_{\mathrm{in}}}{r_{\mathrm{J}}}\right)^{\xi}-\frac{r_{\mathrm{in}}}{r_{\mathrm{J}}}\right]$,

is obtained by computing the difference between the mechanical power that comes in at $r_{\mathrm{J}}$ and goes out at $r_{\mathrm{in}}$. Note that $\dot{M}_{\mathrm{a}, \mathrm{J}}$ is a constant and must be understood here as the accretion rate feeding the JED at $r_{\mathrm{J}}$ (within the JED, the disk accretion rates varies as $\left.\dot{M}_{\mathrm{a}} \propto r^{\xi}\right)$. All available power $P_{\text {acc }}$ is thus shared between radiative losses at the disk surfaces $P_{\text {rad }}$ and jet power $P_{\text {jet }}$. This last term marks the difference with the global energy budget of a SAD. In the latter, all accretion power is released as radiation, whereas JEDs also power jets. This last contribution to the budget is actually the easiest to compute as it is the flux of energy that leaves the two disk surfaces. Namely,

$P_{\text {jet }}=\int\left[\boldsymbol{S}_{\mathrm{MHD}}+\rho \mathcal{E} \boldsymbol{u}_{\mathrm{p}}\right] \cdot \mathrm{d} \boldsymbol{S}$

where $\mathcal{E}=u^{2} / 2+\Phi_{\mathrm{G}}+H$ is the nonmagnetic specific energy $\left(\Phi_{\mathrm{G}}\right.$ is the gravitational potential and $H$ the enthalpy), $S_{\mathrm{MHD}}=-\Omega_{\mathrm{F}} r B_{\phi} \boldsymbol{B}_{\mathrm{p}} / \mu_{\mathrm{o}}$ is the MHD Poynting vector $\left(\Omega_{\mathrm{F}} \simeq \Omega_{\mathrm{K}}\right.$ is the angular velocity of the magnetic field line) and $\mathrm{d} \boldsymbol{S}=\mathrm{d} \boldsymbol{S} \boldsymbol{n}$ with $\mathrm{d} S$ the elementary disk surface and $\boldsymbol{n}$ the unit vector normal to the disk surface. For magnetically driven jets from Keplerian accretion disks, the dominant term is the magnetic contribution due to the MHD Poynting vector. Thus, the energy flux carried away by the jets depends directly on the toroidal field at the disk surface $B_{\phi}^{+}$and is written

$\frac{2 P_{\text {jet }}}{P_{\text {acc }}}=\frac{\Lambda}{1+\Lambda}\left|\frac{B_{\phi}^{+}}{q B_{z}}\right|$

where $\Lambda \sim 1 / \epsilon \gg 1$ is the ratio of the jet to viscous torques, and $q$ the magnetic shear parameter. Most MAES solutions were found with $\left|B_{\phi}^{+}\right| \simeq q B_{z}$, which translates into $P_{\text {acc }} \simeq 2 P_{\text {jet }}$. Thus, most available power feeds the jets and energy conservation reveals that only a fraction of it, namely

$\frac{2 P_{\text {rad }}}{P_{\text {acc }}} \simeq \frac{1}{1+\Lambda} \simeq \varepsilon$

is dissipated within the JED. This justifies the use of Eq. (9). Note, however, some solutions were found with $\left|B_{\phi}^{+}\right| / q B_{z}$ as low as 0.5 , which shows that JEDs may also, under certain circumstances, produce some luminosity. But as a first step to illustrate the effect of JEDs in the centre of YSO accretion disks, we have disregarded these peculiar solutions (see also discussion in Ferreira et al. 2006b).

\section{Appendix B: JED key quantities}

In this appendix, we give the detailed analytical expressions of the main disk quantities as calculated in the MAES context. We only focus on the radial dependence of each quantity. In these expressions, the Rosseland mean opacity is taken under the form $\kappa=\bar{\kappa} \rho^{a} T^{b}$, where the parameters $\bar{\kappa}, a$ and $b$ are to be determined depending on the considered opacity regime. In the following, $\sigma$ is the Stefan-Boltzmann constant; $M_{\star}$ and $\dot{M}_{\text {a }}$ respectively represent the mass of the central object and the mass accretion rate; $\bar{\mu}=2$ (Papaloizou \& Terquem 1999) is the mean molecular weight of the gas; $m_{\mathrm{p}}$ the proton mass and $k_{\mathrm{B}}$ the Boltzmann constant. The parameter $m_{\mathrm{s}}$ is related to the MAES model (see
Sect. 2) and is found to be close to unity in most solutions of the problem. Using Eqs. (11) and (12) to rewrite Eq. (13) from Eqs. (8) and (10), the radial dependence of the midplane temperature is expressed as

$T_{0}(r)=\mathcal{A}_{T} r^{-\Gamma[1+5(a+1) / 2]}$,

where $\Gamma \equiv 1 /(4+a-b)$ and $\mathcal{A}_{T}$ is dependent of the parameters of the problem,

$\mathcal{A}_{T}=\left(\frac{\bar{\kappa}}{8 \pi \sigma}\right)^{\Gamma}\left(\frac{1}{4 \pi m_{\mathrm{s}}}\right)^{\Gamma(a+1)}\left(G M_{\star}\right)^{\Gamma(a+1) / 2} \dot{M}_{\mathrm{a}}^{\Gamma(a+2)}\left(\frac{\bar{\mu} m_{\mathrm{p}}}{k_{\mathrm{B}}}\right)^{a \Gamma}$.

Plugging Eq. (B.1) into Eq. (11), the disk aspect ratio $\epsilon=h / r$ is then directly obtained under the form

$\epsilon(r)=\mathcal{A}_{\epsilon} r^{\frac{1-\Gamma}{2}-\frac{5 \Gamma(a+1)}{4}}$,

$\mathcal{A}_{\epsilon}=\left(\frac{\bar{\kappa}}{8 \pi \sigma}\right)^{\Gamma / 2}\left(\frac{1}{4 \pi m_{\mathrm{s}}}\right)^{\frac{\Gamma(a+1)}{2}}\left(G M_{\star}\right)^{\frac{\Gamma(a+1)}{4}-\frac{1}{2}} \dot{M}_{\mathrm{a}}^{\frac{\Gamma(a+2)}{2}}\left(\frac{\bar{\mu} m_{\mathrm{p}}}{k_{\mathrm{B}}}\right)^{\frac{a \Gamma}{2}-\frac{1}{2}}$.

The disk mass density can be calculated in a similar fashion, (using Eqs. (B.2) and (12) together), but we give the expression of the surface density as it appears to be a more relevant quantity regarding accretion disk studies. The expression of $\Sigma(r)$ is calculated using $\Sigma(r)=\int_{-h}^{+h} \rho(r, z) \mathrm{d} z \sim 2 \rho_{0} h=2 \rho_{0}(r) \epsilon(r) r$, and reads

$\Sigma(r)=\mathcal{A}_{\Sigma} r^{\frac{\Gamma}{2}+\frac{5 \Gamma(a+1)}{4}-1}$,

with

$\mathcal{A}_{\Sigma}=\left(\frac{\bar{\kappa}}{8 \pi \sigma}\right)^{-\Gamma}\left(\frac{1}{4 \pi m_{\mathrm{s}}}\right)^{1-\frac{\Gamma(a+1)}{2}}\left(G M_{\star}\right)^{\frac{-\Gamma(a+1)}{4}} \dot{M}_{\mathrm{a}}^{1-\frac{\Gamma(a+2)}{2}}\left(\frac{\bar{\mu} m_{\mathrm{p}}}{k_{\mathrm{B}}}\right)^{\frac{1}{2}-\frac{a \Gamma}{2}}$.

\section{References}

Alexander, D. R., \& Ferguson, J. W. 1994, ApJ, 437, 879

Alexander, R. D., \& Armitage, P. J. 2006, ApJ, 639, L83

Armitage, P. J. 2007, ArXiv Astrophysics e-prints

Bacciotti, F., Ray, T. P., Mundt, R., Eislöffel, J., \& Solf, J. 2002, ApJ, 576, 222

Balbus, S. A., \& Hawley, J. F. 1991, ApJ, 376, 214

Bally, J., Reipurth, B., \& Davis, C. J. 2007, Protostars and Planets V, 215

Banerjee, R., \& Pudritz, R. E. 2006, ApJ, 641, 949

Barge, P., \& Sommeria, J. 1995, A\&A, 295, L1

Basu, S., \& Mouschovias, T. C. 1994, ApJ, 432, 720

Bell, K. R., \& Lin, D. N. C. 1994, ApJ, 427, 987

Blandford, R. D., \& Payne, D. G. 1982, MNRAS, 199, 883

Bouvier, J., Alencar, S. H. P., Harries, T. J., Johns-Krull, C. M., \& Romanova,

M. M. 2007, in Protostars and Planets V, ed. B. Reipurth, D. Jewitt, \& K. Keil, 479

Calvet, N., D’Alessio, P., Hartmann, L., et al. 2002, ApJ, 568, 1008

Calvet, N., Muzerolle, J., Briceño, C., et al. 2004, AJ, 128, 1294

Casse, F., \& Ferreira, J. 2000a, A\&A, 353, 1115

Casse, F., \& Ferreira, J. 2000b, A\&A, 361, 1178

Casse, F., \& Keppens, R. 2002, ApJ, 581, 988

Casse, F., \& Keppens, R. 2004, ApJ, 601, 90

Caunt, S. E., \& Tagger, M. 2001, A\&A, 367, 1095

Chiang, E. I., \& Goldreich, P. 1997, ApJ, 490, 368

Ciesla, F. J. 2007, ApJ, 654, L159

Clarke, C. J., \& Pringle, J. E. 2006, MNRAS, 370, L10

Crutcher, R. M. 1999, ApJ, 520, 706

D’Alessio, P., Canto, J., Calvet, N., \& Lizano, S. 1998, ApJ, 500, 411

D’Alessio, P., Hartmann, L., Calvet, N., et al. 2005, ApJ, 621, 461

Donati, J.-F., Paletou, F., Bouvier, J., \& Ferreira, J. 2005, Nature, 438, 466

Dubus, G., Lasota, J.-P., Hameury, J.-M., \& Charles, P. 1999, MNRAS, 303, 139

Dullemond, C. P., Dominik, C., \& Natta, A. 2001, ApJ, 560, 957

Dullemond, C. P., Natta, A., \& Testi, L. 2006, ApJ, 645, L69

Dullemond, C. P., Hollenbach, D., Kamp, I., \& D'Alessio, P. 2007, in Protostars and Planets V, ed. B. Reipurth, D. Jewitt, \& K. Keil, 555-572 
Ferrari, A. 1998, ARA\&A, 36, 539

Ferreira, J. 1997, A\&A, 319, 340

Ferreira, J. 2002, Theory of magnetized accretion discs driving jets (EDP Sciences), 229 [arXiv: astro-ph/0311621]

Ferreira, J., \& Casse, F. 2004, ApJ, 601, L139

Ferreira, J., \& Pelletier, G. 1993, A\&A, 276, 625

Ferreira, J., \& Pelletier, G. 1995, A\&A, 295, 807

Ferreira, J., Dougados, C., \& Cabrit, S. 2006a, A\&A, 453, 785

Ferreira, J., Petrucci, P.-O., Henri, G., Saugé, L., \& Pelletier, G. 2006b, A\&A, 447,813

Frank, J., King, A., \& Raine, D. J. 2002, Accretion Power in Astrophysics, third edition ed. J. Frank, A. King, D. Raine (Cambridge, UK: Cambridge University Press), 398

Fromang, S., \& Papaloizou, J. 2006, A\&A, 452, 751

Gammie, C. F. 1996, ApJ, 457, 355

Goldreich, P., \& Tremaine, S. 1980, ApJ, 241, 425

Gullbring, E., Hartmann, L., Briceno, C., \& Calvet, N. 1998, ApJ, 492, 323

Hartmann, L., D’Alessio, P., Calvet, N., \& Muzerolle, J. 2006, ApJ, 648, 484

Heiles, C., Goodman, A. A., McKee, C. F., \& Zweibel, E. G. 1993, in Protostars and Planets III, ed. E. H. Levy \& J. I. Lunine, 279

Henning, T., \& Stognienko, R. 1996, A\&A, 311, 291

Heyvaerts, J., Priest, E. R., \& Bardou, A. 1996, ApJ, 473, 403

Hubeny, I. 1990, ApJ, 351, 632

Keppens, R., Casse, F., \& Goedbloed, J. P. 2002, ApJ, 569, L121

Kuncic, Z., \& Bicknell, G. V. 2007, Mod. Phys. Lett. A, 22, 1685

Masset, F. S., Morbidelli, A., Crida, A., \& Ferreira, J. 2006, ApJ, 642, 478

Ménard, F., \& Duchêne, G. 2004, A\&A, 425, 973
Mirabel, I. F., \& Rodríguez, L. F. 1999, ARA\&A, 37, 409

Muzerolle, J., Hillenbrand, L., Calvet, N., Briceño, C., \& Hartmann, L. 2003, ApJ, 592, 266

Najita, J. R., Carr, J. S., Glassgold, A. E., \& Valenti, J. 2007, ArXiv e-prints, 704 Padoan, P., Kritsuk, A., Norman, M. L., \& Nordlund, A. 2005, ApJ, 622, L61 Papaloizou, J. C. B., \& Terquem, C. 1999, ApJ, 521, 823

Pesenti, N., Dougados, C., Cabrit, S., et al. 2004, A\&A, 416, L9

Pringle, J. E. 1981, ARA\&A, 19, 137

Pringle, J. E., \& Rees, M. J. 1972, A\&A, 21, 1

Pudritz, R. E., \& Norman, C. A. 1986, ApJ, 301, 571

Rafikov, R. R., \& De Colle, F. 2006, ApJ, 646, 275

Ratzka, T., Leinert, C., Henning, T., et al. 2007, A\&A, 471, 173

Sauty, C., Trussoni, E., \& Tsinganos, K. 2002, A\&A, 389, 1068

Semenov, D., Henning, T., Helling, C., Ilgner, M., \& Sedlmayr, E. 2003, A\&A, 410,611

Shakura, N. I., \& Sunyaev, R. A. 1973, A\&A, 24, 337

Shu, F. H., Najita, J. R., Shang, H., \& Li, Z.-Y. 2000, Protostars and Planets IV, 789

Stehle, R., \& Spruit, H. C. 2001, MNRAS, 323, 587

Strom, K. M., Strom, S. E., Wolff, S. C., Morgan, J., \& Wenz, M. 1986, ApJS, 62,39

Tagger, M., \& Pellat, R. 1999, A\&A, 349, 1003

Tagger, M., Pellat, R., \& Coroniti, F. V. 1992, ApJ, 393, 708

Wardle, M. \& Koenigl, A. 1993, ApJ, 410, 218

Whelan, E. T., Ray, T. P., Bacciotti, F., et al. 2005, Nature, 435, 652

Zanni, C., Ferrari, A., Rosner, R., Bodo, G., \& Massaglia, S. 2007, A\&A, 469, 811 